\title{
A Two-Electrode 2.88nJ/Conversion Biopotential Acquisition System for Portable Healthcare Device
}

\author{
Long Yan, Namjun Cho, Jerald Yoo, Binhee Kim and Hoi-Jun Yoo \\ Department of EECS, Korea Advanced Institute of Science and Technology (KAIST) \\ 373-1, Guseong-dong, Yuseong-gu \\ Daejeon, 305-701, Republic of Korea \\ Email: yanlong@eeinfo.kaist.ac.kr
}

\begin{abstract}
A $2.88 \mathrm{~nJ} /$ Conversion low energy biopotential acquisition system is designed for portable healthcare device. Two dry copper contact electrodes with $1.2-\mathrm{cm}$ diameter are used to easily interface between skin and healthcare device. Chopping technique is adopted at readout front end to obtain thermal noise floor of $1.3 \mathrm{uVrms}$ over $0.5 \sim 200 \mathrm{~Hz}$ and CMRR over $100 \mathrm{~dB}$ to mitigate common-mode body potential induced from $\mathrm{AC}$ power line. A 4-stage gain control and band selection blocks are integrated to digitally calibrate for different types of biomedical signal and an 8-bit synchronous successive approximation register (SAR) A/D is used to digitize sensed biopotentials. A test chip is implemented in 0.18um, 1.8V supply CMOS technology and successively verified by readout ECG signal with two electrodes contact at chest of body with separating $6 \mathrm{~cm}$.
\end{abstract}

\section{INTRODUCTION}

ECG, EMG and EEG are common biomedical signals that give continuous information for patients undergoing surgery or those admitted to intensive care units (ICUs). Conventionally, patients are measured by bulky and mainpowered instrument with several wired leads connected on body which can greatly reduce patient's mobility and white coat effect in hospital also makes an erroneous diagnosis. Common portable health care approaches which use three $\mathrm{Ag} / \mathrm{AgCl}$ electrodes with conductive solution provide good signal quality in Skin-Electrode interface [1]. However, it has the problem of inconvenient patient attachment; also it may cause inflammation of the human skin and limits its reusability and continuous health monitoring. Furthermore, the third reference electrode forms a low impedance node to ground. Hence, patients suffer from hazard of leakage current. Consequently, a low energy, small form factor, two dry electrodes biopotential acquisition system is attractive for ambulatory healthcare application. Previously, several types of commercial metals were investigated as a wearable electrode [2]. The impedance fluctuation of the different metal electrodes is less than one decade in the frequency range less than $1 \mathrm{kHz}$ compared with $\mathrm{Ag} / \mathrm{AgCl}$. This indicates that a

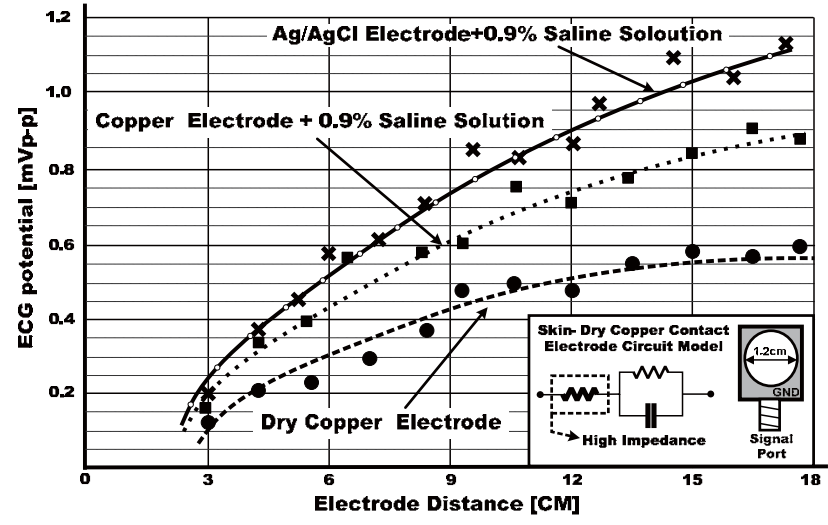

Fig. 1 Electrode comparison between conventional $\mathrm{Ag} / \mathrm{AgCl}$ with saline solution and dry PCB type copper contact electrode

copper on $\mathrm{PCB}$ as an interface between skin and portable instrument can replace the $\mathrm{Ag} / \mathrm{AgCl}$ electrode without any electrically conductive solution. However, its main drawback is that power line interference problem is severe in twoelectrode recording system [3][4]. Short distance between two electrodes can reduce electromagnetic interference (EMI) in biopotential measurements since this approach will cause power line $60 \mathrm{~Hz}$ noise common to readout front end. However, it also suppresses differential signal strength because of the short distance between the electrodes. Moreover, due to the lack of the conductive solution, the skin-electrode interface impedance of dry electrode is much larger than conventional $\mathrm{Ag} / \mathrm{AgCl}$ electrode which could also degrade signal potential as shown in Fig.1. Therefore, in order to extract biopotenials under such circumstances, the noise and CMRR requirements of analog readout front end are more stringent, while consuming low power. According to [4], only a micro volts noise level is allowed to maintain recorded signal quality at the front end and over 100dB in band CMRR and PSRR of readout front end are also required for robust operation regards to power line interference in such circumstances. Simultaneously, the biopotential acquisition system need to have a programmability for its gain and bandwidth [5] to meet different signal potential and frequency characteristics. 


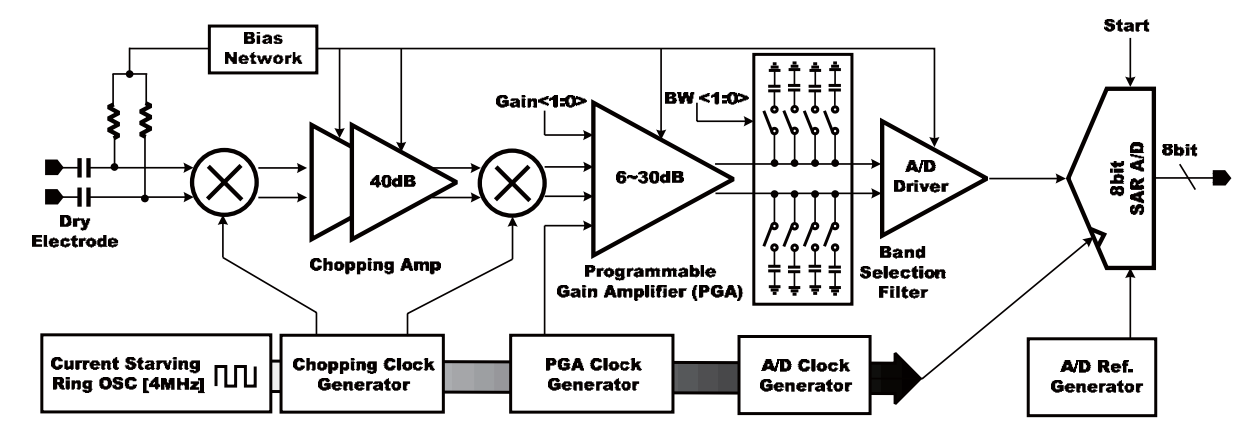

Fig. 2 The Proposed Architecture of Low Noise, Low Energy, Two-Electrode Biomedical Signal Acquisition System

In this paper, the proposed architecture of a low noise, low energy, two dry electrodes biopotential acquisition system is described in Section II. In Section III, The building blocks including low noise, micro power chopping amplifier, programmable gain and band selection stage, low energy 8-bit Synchronous SAR A/D are discussed. In Section IV, Measurement results are presented. Finally, the conclusion is given.

\section{The Proposed Architecture of Two-Electrode BIOPOTENTIAL ACQUISITION SYSTEM}

Fig. 2 shows the Architecture of the proposed low noise, low energy, two-electrode biopotential acquisition system. It mainly consists of Bio-Amp and A/D. The sensed biomedical signal through copper contact electrode firstly DC coupled and low noise pre-amplified by Bio-Amp while consuming minimum power. Low energy consumption could be achieved through the 8-bit SAR A/D which can continuously digitize the sensed biopotenial in high sampling rate. Bio-Amp includes low noise chopping amplifier (Amp.) cascaded by switched capacitor (SC) type digitally programmable gain and bandwidth selection stage. KT/C noise can be minimally referred to input stage by allocating $40 \mathrm{~dB}$ in-band gain to chopping stage. Finally, a $1 \mathrm{x} \mathrm{A} / \mathrm{D}$ driver is operated as a differential to single-ended converter to interface between Bio-Amp and A/D. SAR A/D is designed by 8-bit binary weighted capacitor type D/A with unit capacitor of $30 \mathrm{fF}$ and a low power dynamic comparator with 8-bit unified sequencer and code register type SAR logic. An On-chip 4MHz current starving ring oscillator with variable frequency divider is integrated to provide different types of frequency including chopping frequency of $7.8 \mathrm{kHz}$, a frequency of $125 \mathrm{kHz}$ as a PGA clock to switch capacitor and a programmable ADC sampling clock for operating SAR A/D. The bias network generates various bias voltages for Bio-Amp block and a design [6] based on resistively weighted difference between the gate-source voltages of NMOS and PMOS as the A/D reference.

\section{BUILDING BLOCKS OF THE BIO-AMP}

\section{A. Chopping Amplifier}

In two electrodes recording system, the principle interference contribution is due to the displacement current from AC power line induced into the patient body. This leads to the total input-referred interference voltage as

$$
V_{i}=V_{i D}+V_{i C}\left(\frac{1}{C M R R}+\left\{\frac{Z_{C M}}{Z_{C M}+Z_{i}}-\frac{Z_{C M}}{Z_{C M}+Z_{i}+\Delta Z_{i}}\right\}\right)+V_{i N}
$$

Where total input-referred interference voltage $V_{i}$ can be expressed in terms of common mode and differential mode voltage at readout front end's input $V_{i C}$ and $V_{i D}$ which produced by the displacement current. $Z_{C M}$ represents input common-mode impedance of amplifier and $Z_{i}, \Delta Z_{i}$ represent skin-electrode interface impedance and it's mismatch. $V_{i N}$ is input-referred offset voltage generated by the front end circuit. Thanks to PCB fabrication technology, we can minimize $\Delta Z_{i}$ to reduce loading effect of high impedance electrode. Analog chopping technique [7] is adopted to dynamically improve CMRR and PSRR and minimize low frequency noise caused by front end circuit. Fig. 3 shows proposed low noise, low power chopping Amp topology. The input chopper modulates input signal to chopping frequency and after two-

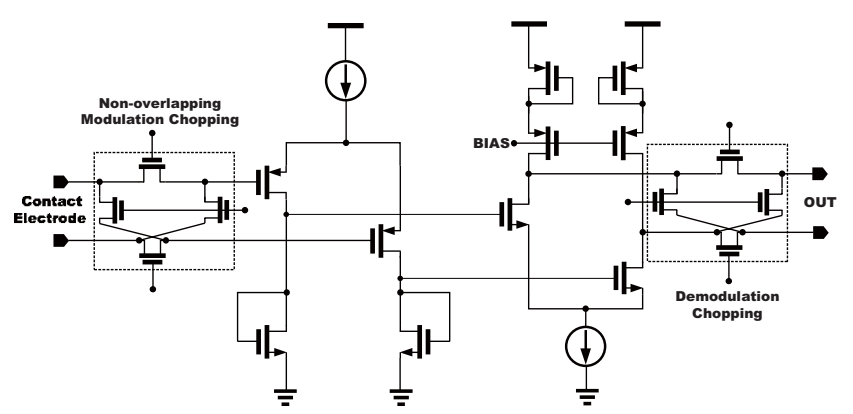

Fig. 3 Low Power Chopping Amplifier 
stage amplification then demodulated, while offset voltage is modulated to chopping frequency and then low pass filtered by simple $\mathrm{Gm}-\mathrm{C}$ filter. Two-stage amplification extends the bandwidth of chopping amplifier to reduce residual offset when demodulated. This stage provides $40 \mathrm{~dB}$ DC gain and its thermal noise floor of $40 \mathrm{nV} / \mathrm{srtHz}$ while consuming only $2 \mathrm{uA}$ from $1.8 \mathrm{~V}$ supply voltage. The noise efficiency factor introduced in (2)[8] shows 5.1 over $200 \mathrm{~Hz}$ bandwidth.

$$
N E F=V_{n i, r m s} \sqrt{\frac{2 I_{\text {total }}}{\pi \cdot V_{t} \cdot 4 k T \cdot B W}}
$$

\section{B. Programmable Gain and Band Selection Circuits}

Fig.4 shows Pseudo-Differential programmable gain and band selection circuits. The Programmable Gain Stage organizes binary weighted capacitor to configure the Bio-Amp gain. This design provides a variable $\mathrm{DC}$ gain from $6 \mathrm{~dB} \sim 30 \mathrm{~dB}$ and $10 \mathrm{pF}$ internal miller capacitor is nested to suppress switching glitches to improve signal quality. Band selection filter is designed by switching load capacitor to set cutoff frequency of $300 \mathrm{~Hz}, 1 \mathrm{kHz}, 10 \mathrm{kHz}$. Finally a $1 \mathrm{x}$ buffer stage is added to drive $\mathrm{A} / \mathrm{D}$.

\section{Low Energy Fully Synchronous SAR A/D}

Low Energy 8-bit SAR A/D with unified sequencer and code resister SAR logic is shown in Fig.5.and Fig.6. Both Delta-Sigma and successive approximation A/D are well suited to meet the low power consumption and optimized for operation at low speed required biomedical application. However, SAR A/D which is shown in Fig.5 superior to Delta-Sigma architecture in simple hardware required and minimal analog blocks to achieve low energy consumption at the same sampling rate. Most common design of the SAR logic is separated sequencer code register architecture [9]. Despite of its simplicity and easy layout which consist of identical $16 \mathrm{~F} / \mathrm{F}$ to provide 8-bit SAR, it's successive approximation algorithm is realized by sequencer synchronously control the code register. Then the code register operates asynchronously control the digital inputs to binary capacitor array since the clock input to a particular $\mathrm{F} / \mathrm{F}$ is obtained from the output of the succeeding F/F. Fig.6 shows unified sequencer and code register SAR logic. In this design a single Set/Reset $\mathrm{F} / \mathrm{F}$ is used in each bit cell which can functions both sequencer and code register. Consequently, Only $8 \mathrm{~F} / \mathrm{F}$ was required and it operates synchronously to

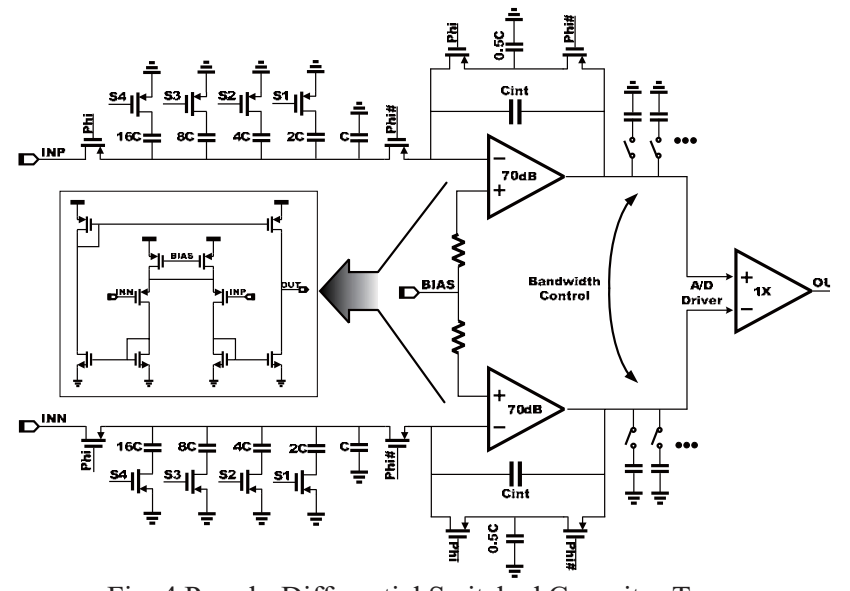

Fig. 4 Pseudo-Differential Switched Capacitor Type Programmable Gain and Band Selection Circuits

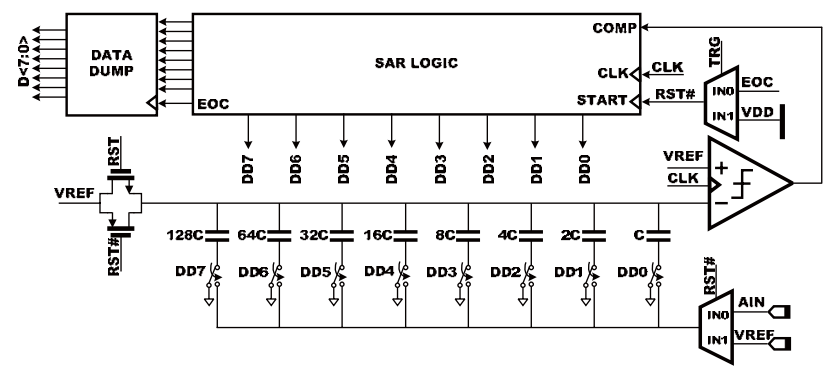

Fig. 5 8-bit low energy fully synchronous SAR A/D

complete successive approximation algorithm. Two standard $\mathrm{F} / \mathrm{F}$ is added to generate End of Conversion (EOC) signal to parallelize the 8-bit output code.

\section{Proposed System Measurement Results}

The proposed biopotential acquisition system was tested by using simple two PCB copper pads as the electrodes with the distance of $6 \mathrm{~cm}$ to sensing ECG signal. Fig.7 shows Amplified ECG waveform by two-electrode Bio-Amp with the gain of $60 \mathrm{~dB}$ and bandwidth of $300 \mathrm{~Hz}$. A TI 3-electrode simplified ECG circuit introduced in [10] is also integrated on the same test board to compare the sensed signal quality. It shows that proposed two-electrode Bio-Amp clearly track the PQRST information of ECG signal.

Fig.8 shows FFT results of 8-bit SAR A/D. The measured SNDR of $47.5 \mathrm{~dB}$ with the sine input frequency of $1 \mathrm{kHz}$ and $400 \mathrm{mVp}-\mathrm{p}$ while consuming only 4.2uW.Table I summarizes the performance of the proposed system. The proposed system was fabricated in 0.18 um CMOS process and its die area of

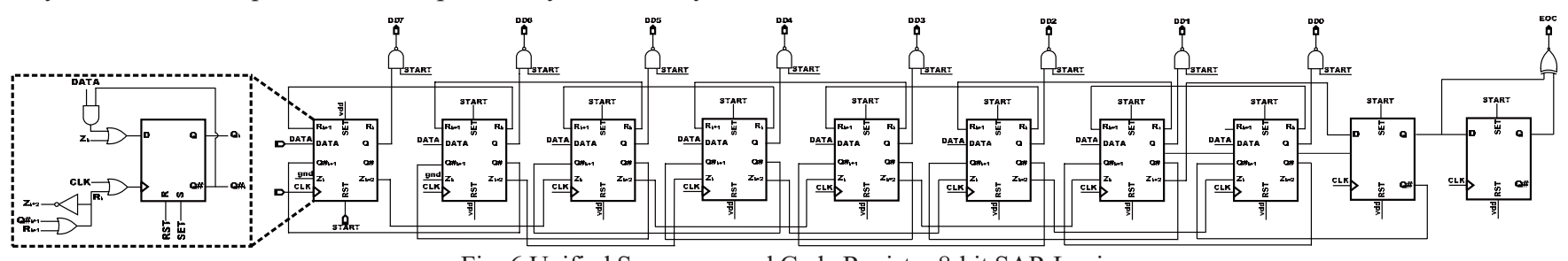

Fig. 6 Unified Sequencer and Code Register 8-bit SAR Logic 
$1.2 \mathrm{~mm} \times 2 \mathrm{~mm}$ as shown in Fig. 9.

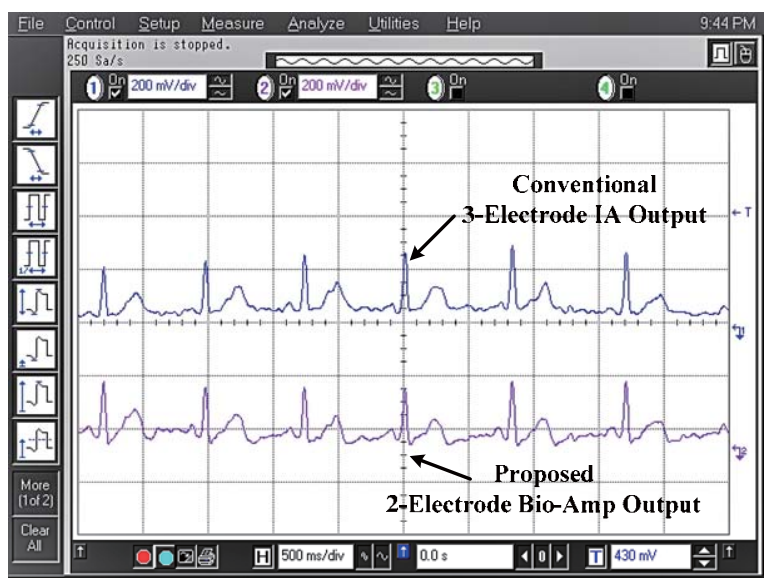

Fig.7. Measured ECG Signal at human chest

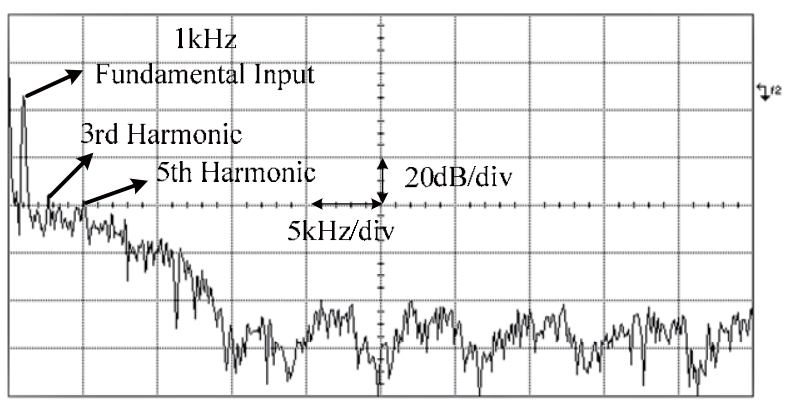

Fig.8. FFT Results of SAR A/D with $1 \mathrm{kHz}$ input

\section{CONCLUSIONS}

We propose a Two-Electrode $2.88 \mathrm{~nJ} /$ conversion biopotential acquisition system which is suited for energy constrained portable healthcare device. Two copper contact pads are used for signal acquisition to achieve mobile health monitoring. Chopping technique was adopted to compensate copper electrodes imperfection and reduce power line interference. The total power consumption of the system is $40 \mathrm{uW}$ with the operation of $125 \mathrm{kHz}$ to yield $2.88 \mathrm{~nJ} /$ Sample. The chip was verified by successively recording ECG signal with separating two copper contact pads of $6 \mathrm{~cm}$.

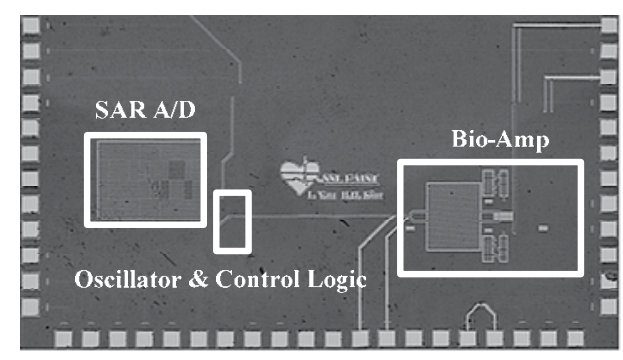

Fig. 9 Chip micro-photograph
TABLE 1

Performance Summary of Biopotential Acquisition System

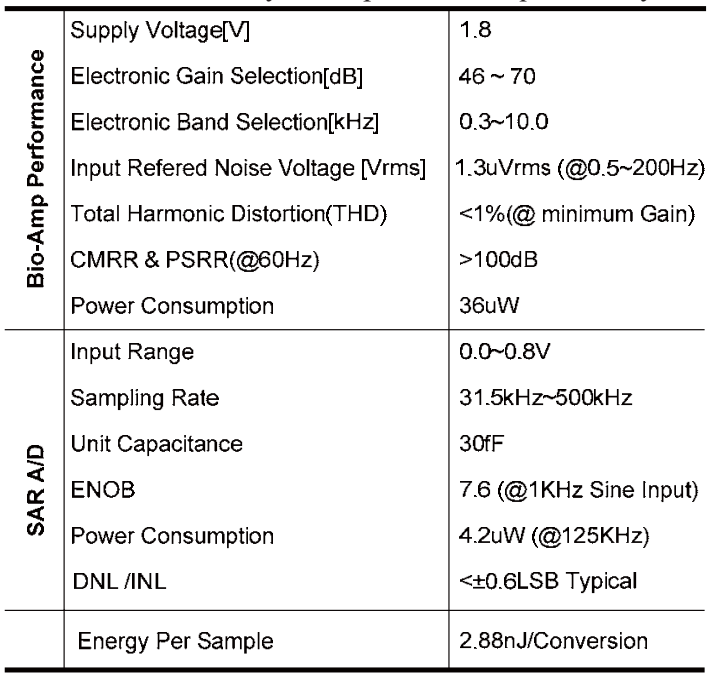

\section{REFERENCES}

[1] John G. Webster, "Medical Instrumentation: Application and Design," $3^{\text {rd }}$ ed.,pp183 205,Wiley.1998

[2] K. Hachisuka, A. Nakata, T. Takeda, Y. Terauchi, K. Shiba, K. Sasaki,H. Hosaka, and K. Itao, "Development and performance analysis of anintra-body communication device," in Int. SolidState Sens., Actuators, Microsyst. Conf., Jun. 2003, pp. 17221725

[3] Enrique M. Spinelli and Miguel A. Mayosky, "Two-Electrode biopotential measurements: Power line interfere Analysis," IEEE Transaction on biomedical engineering, vol.52, pp. 1436442, Aug. 2005.

[4] D.E.Wood, D.J.Ewins, and W.Balachandran, "Comparative analysis if powr line interference between two-or three-electrode biopotential amplifiers," Medical \& Biological Engineering \& Computing, vol.33, pp.63-68, 1995.

[5] Refet Firat Yazicioglu, Chris Van Hoff,et.al.,"A 60uW $60 \mathrm{nV} / \mathrm{srtHz}$ Redout Front-End for Portable Biopotential Acquisition Systems," IEEE Journal of Solid-State Circuits, vol.42, pp.1100-1109, May.2007.

[6] K.N.Leung and P.K.T.Mok, "A CMOS Voltage Reference based pm weighted $\Delta \mathrm{V}_{\mathrm{GS}}$ for CMOS Low-Dropout Linear Regulators," IEEE Journal of Solid-State Circuits, vol.38, pp.146-150,Jan.2003.

[7] Christian C. ENZ and Gabor C. Temes"Circuit Techniques for Reducing the Effects of Op-Amp Imperfections: Autozeroing, Correlated Double Sampling, and Chopper Stablization," Proc. IEEE, vol. 84, pp. 1584-1614,Nov. 1996.

[8] M.S.J Steyaert,W.M.C Sansen,et.al.,"Amicropower low-noise monolithic instrumentation amplifier for medical purposes," IEEE Journal of Solid-State Circuits, vol.SC-22, pp.11631168,1986

[9] You-Kuang Chang, et. Al., “A 8-bit 500-KS/s Low Power SAR ADC for Bio Medical Applications," IEEE Asian Solide-State Circuits Conference, pp.228-231,Nov.2007.

[10] http://focus.ti.com/lit/ds/sbos168d/sbos168d.pdf 